\title{
Mirando sobre y bajo el agua
}

\author{
Looking over and under water \\ Juan Francisco Trevizan Rispoli
}

\begin{abstract}
“..... Los ríos son nuestros hermanos, ellos calman nuestra sed. Los ríos llevan nuestras canoas y alimentan a nuestros hijos. Si os vendemos nuestras tierras, deberéis recordar y enseñar a vuestros hijos que los ríos son nuestros hermanos y hermanos de vosotros; deberéis en adelante dar a los ríos el trato bondadoso que daréis a cualquier hermano...".

Noah Sealth (1786-1866), jefe de la tribu de los Suquamish
\end{abstract}

"La humanidad tiene la capacidad de hacer desarrollo sostenible, asegurando las necesidades del presente, sin comprometer la capacidad de las futuras generaciones de satisfacer sus propias necesidades". Brundtland $1987^{1}$

\section{RESUMEN}

El agua es un compuesto que gracias a sus propiedades ha posibilitado la vida, ha conformado el paisaje y ha moldeado las civilizaciones en la faz de la tierra. No todas las regiones poseen la misma disponibilidad y su escasez relativa; junto con el cambio climático está generando conflictos entre pueblos y actividades económicas y medioambientales, pero siendo un conflicto global, se debe enfrentar en la esfera local, para lo cual se deben fortalecer las capacidades locales en todos aquellos ámbitos en que el agua participa.

Palabras clave: Recursos hídricos, sustentabilidad, cambio climático, medioambiente.

\section{ABSTRACT}

Thanks to the properties of water life is possible; it has shaped the landscape and molded the civilizations on the face of the earth. Not all regions have adequate availability of water; its relative scarcity together with climate change is generating conflicts between nations and between economic activity and environmental concerns. Although this is a global conflict, it must be faced in local spheres, thus it is necessary to strengthen local capacities in all areas in which water is a factor.

Key words: Water resources, sustainability, climate change, environment.

\section{El agua en el planeta}

El agua es uno de los compuestos químicos cuyas propiedades han posibilitado el desarrollo de la vida en nuestro planeta. Sus propiedades especiales, tales como el calor específico, calor de vaporización, su conductividad eléctrica, calor de fusión, tensión superficial, viscosidad, las variaciones en su densidad, su capacidad como disolvente universal, su capacidad para participar en las distintas reacciones químicas y su carácter anfótero, hacen que el agua esté presente en casi todos los procesos, participando o posibilitando que ellos se produzcan. No en vano nuestro cuerpo está en dos tercios aproximadamente compuesto por agua y se nos dificulta la vida después de 3 o 4 días

\footnotetext{
Ingeniero agrónomo. Universidad de Tarapacá. Arica-Chile. E-mail: jftrevizan@gmail.com

Informe Brundtland. Informe socioeconómico elaborado por especialistas de distintas naciones en 1987 para la ONU, por una comisión encabezada por la doctora Gro Harlem Brundtland. Originalmente, se llamó Nuestro Futuro Común (Our Common Future, en inglés). En este informe, se utilizó por primera vez el término desarrollo sostenible (o desarrollo sustentable), definido como aquel que satisface las necesidades del presente sin comprometer las necesidades de las futuras generaciones. Implica un cambio muy importante en cuanto a la idea de sustentabilidad, principalmente ecológica, y a un marco que da también énfasis al contexto económico y social del desarrollo(8)
} 
sin beberla. Mucho hemos aprendido del agua y su comportamiento, desde Lewis (1923) y su teoría de los enlaces covalentes hasta nuestros días, sin embargo no cabe duda que existe una infinidad de cosas por aprender.

El agua del planeta se estima en 1.373 trillones de litros. La mayor parte de esta, un 97,5\%, se encuentra en los océanos, en los glaciares y casquetes polares un $1,74 \%$; los acuíferos, la verdadera reserva para el hombre, un $0,76 \%$. Los lagos encierran el $0,007 \%$, mientras que la cifra desciende en los mares interiores a un $0,006 \%$. La biomasa mantiene el $0,0001 \%$, la atmósfera el $0,001 \%$ y los ríos tan sólo $0,0002 \%$ del total. Esta cantidad ha estado circulando siempre por la Tierra, originando y conservando la vida en ella. Disponemos actualmente de la misma cantidad de la que disfrutaban los dinosaurios hace 65 millones de años.

Sin embargo, el agua no es un compuesto que se mantiene estático en la naturaleza, ya que a partir de sus propiedades que le permiten pasar de estado sólido a líquido y gaseoso, y viceversa, y "colarse" por cada rincón del planeta le permiten mantenerse en un eterno movimiento que conocemos como ciclo del agua o ciclo hidrológico.

El ciclo hidrológico empieza con la evaporación del agua desde la superficie de los océanos o de otras fuentes de agua libre. A medida que se eleva (evaporación) el aire humedecido se enfría y el vapor se transforma en agua (condensación), las gotas se juntan y forman una nube, para luego caer por su propio peso (precipitación).

Una parte del agua que llega a la superficie terrestre será aprovechada por los seres vivos; otra escurrirá por el terreno hasta llegar a un río, un lago o el océano. A este fenómeno se le conoce como escorrentía. Otro porcentaje del agua se filtrará a través del suelo, formando capas de agua subterránea, conocidas como acuíferos. Este proceso es la percolación. Tarde o temprano, esta agua llegará a la superficie y volverá nuevamente a la atmósfera, dando inicio a un nuevo ciclo.

Las distintas masas de agua que se encuentran distribuidas en el planeta y que circulan por este, lo hacen a distintas velocidades y por distintos caminos, algunas rápidas como por los ríos y otras que se estacionan por períodos milenarios en acuíferos profundos. Entonces llamamos tiempo de residencia al tiempo que se demora el agua en permanecer en los distintos lugares de la hidrosfera.
Tabla 1. Tiempo medio de residencia del agua.

\begin{tabular}{ll}
\hline Océanos & 2.500 años \\
\hline Glaciares y casquetes polares & 9.700 años \\
Subterráneas dulces & decenas de miles de años \\
Lagos dulces & 17 años \\
Lagos salados & 150 años \\
Ríos & $15-20$ días \\
Biomasa & algunas horas \\
Atmósfera & 8 a 10 días \\
\hline
\end{tabular}

\section{El agua conforma el paisaje y las civilizaciones}

Las aguas se distribuyen a lo largo y ancho del planeta en forma desigual y junto con otras variables como la temperatura, la altitud, generan distintas zonas ecológicas que van desde desiertos a zonas de bosques húmedos y casquetes polares.

En este escenario las civilizaciones se fueron asentando y desarrollando en aquellos lugares en donde el agua fluía con abundancia relativa, el Éufrates, el Nilo, el río Amarillo, el Po, el Rin, el Danubio, el Sena, el Támesis, el río Tijuana, el Colorado, el Misisipi, el río Grande, el Paraná, el Beni, entre tantos dan cuenta de esto. Sin embargo, no solo los grandes ríos han cobijado a los pueblos; para quien vive en el norte de Chile, impresiona ver cómo se asentaron poblaciones humanas en pequeñas cuencas con vertientes minúsculas que lograban satisfacer las necesidades locales.

A medida que las sociedades se fueron haciendo más complejas, sus conocimientos fueron sirviendo para el manejo de los recursos naturales y el agua fue conducida a aquellos lugares donde se requería (y nos maravillamos hasta hoy día con los acueductos desplegados por Europa por el imperio Romano, los canales y terrazas de cultivos del imperio Inca, los acueductos Aztecas construidos en la época de Moctezuma, etc.). Pero no sólo eso, sino que también se dieron normas, leyes, organizaciones, formas de resolver conflictos (aquí es muy interesante revisar las historias de las comunidades de agua, algunas como las españolas que tienen más de mil años de funcionamiento). Infraestructura y organizaciones fueron resolviendo los requerimientos de agua potable, riego, para sus procesos industriales y desecho de las aguas servidas.

Pero el agua no está repartida de igual manera por la tierra y a cada país le corresponde su propia realidad con respecto a los recursos hídricos, lo que a medida que aumenta la población hace más patente el problema. 
Tabla 2. Disponibilidad de Recursos Hídricos de algunos países.

\begin{tabular}{|c|c|c|c|}
\hline País & $\begin{array}{l}\text { Población (miles de } \\
\text { habitantes) }\end{array}$ & $\begin{array}{l}\text { Tasa de precipitación } \\
(\mathrm{mm} / \mathrm{año})\end{array}$ & $\begin{array}{l}\text { Rec. Hídricos Totales Renovables } \\
\text { por habitante en el } 2000\left(\mathrm{~m}^{3} / \mathrm{año}\right)\end{array}$ \\
\hline Alemania & 82.526 & 700 & 1.878 \\
\hline Arabia Saudita & 24.919 & 100 & 118 \\
\hline Argentina & 38.871 & 600 & 21.981 \\
\hline Australia & 19.913 & 500 & 25.708 \\
\hline Bahamas & 317 & 1.300 & 66 \\
\hline Bolivia & 8.973 & 1.100 & 74.743 \\
\hline Brasil & 180.654 & 1.800 & 48.314 \\
\hline Chile & 15.996 & 700 & 60.614 \\
\hline China & 1.320 .892 & 600 & 2.259 \\
\hline Colombia & 44.914 & 2.600 & 50.635 \\
\hline Costa Rica & 4.250 & 2.900 & 27.932 \\
\hline Cuba & 11.328 & 1.300 & 3.404 \\
\hline Ecuador & 13.192 & 2.100 & 34.161 \\
\hline Egipto & 73.390 & 100 & 859 \\
\hline El Salvador & 6.614 & 1.700 & 4.024 \\
\hline Emiratos Árabes Unidos & 3.051 & 100 & 58 \\
\hline España & 41.128 & 600 & 2.794 \\
\hline Estados Unidos de América & 297.043 & 700 & 10.837 \\
\hline Francia & 60.434 & 900 & 3.439 \\
\hline Guatemala & 12.661 & 2.700 & 9.773 \\
\hline Haití & 8.437 & 1.400 & 1.723 \\
\hline Honduras & 7.099 & 2.000 & 14949 \\
\hline India & 1.081 .229 & 1.100 & 1.880 \\
\hline Israel & 6.560 & 400 & 276 \\
\hline Japón & 127.800 & 1.700 & 3.383 \\
\hline México & 104.931 & 800 & 4.624 \\
\hline Países Bajos & 16.227 & 800 & 5.736 \\
\hline Perú & 27.567 & 1.500 & 74.546 \\
\hline Somalia & 10.312 & 300 & 1.538 \\
\hline Sudáfrica & 45.214 & 500 & 1.154 \\
\hline Túnez & 9.937 & 300 & 482 \\
\hline
\end{tabular}

\section{El agua y la problemática global}

Lejos están los tiempos en que las poblaciones crecían y seguían colonizando nuevas tierras; hoy la población del mundo es de 6.000 millones y crece a razón de unos 80 millones por año. Esta cifra entraña un aumento de la demanda de agua dulce de aproximadamente 64.000 millones de metros cúbicos por año. Para dar una dimensión de lo galopante de la problemática que se va generando, resalta el hecho que desde 1970 se han añadido al planeta casi 2.000 millones de habitantes, por lo que se dispone ahora de un tercio menos de agua per cápita que entonces. El problema se agrava si se espera que para el año 2050 la población alcance los 9.000 millones, aumentando lo que conocemos como tensión hídrica.

Malin Falkenmark (3) formuló estos conceptos de tensión hídrica y de escasez de agua basándose en un índice de las necesidades de agua dulce per cápita. Para ello estimó una necesidad mínima de 100 litros por día por persona para uso doméstico, y de 5 a 20 veces más para usos agrícolas e industriales. Estos conceptos han sido ampliamente aceptados y empleados por los hidrólogos, el Banco Mundial y otras organizaciones.

Se dice que un país experimenta tensión hídrica cuando el suministro anual de agua desciende a menos de 1.700 metros cúbicos por persona por año, cuando desciende a niveles de 1.700 a 1.000 metros cúbicos por persona, pueden preverse situaciones de escasez periódica o limitada de agua. Cuando los suministros anuales de agua bajan a menos de 1.000 metros cúbicos por persona, el país enfrenta escasez de agua. Una vez que un país experimenta escasez de agua, puede esperar una escasez crónica que amenace la producción de alimentos, obstaculice el desarrollo económico y dañe los ecosistemas. (3)(5) 
Esta desigual disponibilidad de agua entre países también se hace patente al interior de cada uno de ellos, ya que cada una de las regiones tiene su propia distribución interna. En el caso de Chile que es un país de más de $4.000 \mathrm{~km}$ de longitud norte -sur encontramos una situación de desierto por el norte y zonas híper lluviosas por el sur.

Tabla 3. Disponibilidad de agua por regiones en Chile.

\begin{tabular}{lrc}
\hline & \multicolumn{2}{c}{ Indicador de Falkenmark m3/cápita/año } \\
\hline Región & 2000 & 2025 \\
\hline I & 1.280 & 929 \\
II & 311 & 240 \\
III & 656 & 468 \\
IV & 2.452 & 1.841 \\
V & 1.224 & 983 \\
RM & 730 & 544 \\
VI & 8.759 & 6.779 \\
VII & 27.589 & 23.773 \\
VIII & 27.116 & 22.206 \\
IX & 37.551 & 30.814 \\
X & 153.150 & 125.274 \\
XI & 3.362 .822 & 2.508 .208 \\
XII & 2.023 .658 & 1.795 .419 \\
\hline
\end{tabular}

Fuente: DGA (2).

Por otro lado, las distintas actividades que realiza el hombre demandan distintos volúmenes de agua entrando cada vez más en conflicto entre ellas. Sin duda la agricultura es una de las actividades que más hace uso de los recursos hídricos y que genera también importantes situaciones de contaminación, lo que obliga a todos los involucrados en esta actividad a tomar conciencia y aumentar significativamente las prácticas que mejoren su uso. En este contexto la agricultura tendrá que responder a modelos cambiantes de demanda de alimentos y combatir la inseguridad alimentaria y la pobreza en las comunidades marginadas. Al hacer esto, la agricultura deberá competir por la escasa agua disponible con otros usuarios y al mismo tiempo reducir la presión sobre el ambiente. Sin embargo, esto no libera a las otras actividades, como es, por ejemplo la minería, de su deber de tomar el mismo camino.

La situación de escasez relativa, la mala utilización de los recursos, la contaminación, la existencia de recursos compartidos entre países, la asimetría entre las actividades productivas y de consumo humano, la poca claridad de las políticas públicas, el desconocimiento de la
Tabla 4. Uso del agua consuntiva en Chile.

\begin{tabular}{lr}
\hline Riego & $84,50 \%$ \\
Minería & $4,50 \%$ \\
Industria & $6,50 \%$ \\
Agua Potable & $4,50 \%$ \\
\hline
\end{tabular}

Fuente: DGA (2).

población, la apropiación del recurso, los costos para mejorar la oferta, la legislación inapropiada, han ido cada vez mas instalándose en las mesas de discusión.

Hoy el conflicto se hace más patente en las zonas áridas y semiáridas, y muchas veces se escucha "nuestro problema es el agua". Pero como hemos analizado, el agua lejos de ser un problema es uno de los compuestos más maravillosos que se han conjugado en el universo para posibilitar el origen de la vida, y luego, reunir comunidades en torno a ella, posibilitando el desarrollo de civilizaciones humanas; en estos últimos momentos de la historia de nuestro planeta, esperamos que no sea ella la causa de nuestra desaparición de la faz de la tierra.

Nadie puede desconocer el creciente interés mundial por el agua, que desde la década de los 70 con la "Conferencia de Naciones Unidas sobre el Ambiente Humano, Estocolmo 1972” (1) y la "Conferencia de la Naciones Unidas sobre Agua, Mar del Plata, 1977" (6) se ha venido desarrollando, lo que se ha traducido en una larga lista de encuentros, conferencias y distintas instancias para poner en relieve la problemática de tan vital elemento para la vida humana.

Del análisis de los temas de estas reuniones se puede decir que se ha producido un cambio en el enfoque bajo el que se consideró inicialmente. Así se pasa del énfasis en el mejoramiento de la provisión de agua potable y saneamiento básico, que fue el tema principal desde los años 70 a los 90, a una mayor preocupación por la gestión en sí, con énfasis en cuestiones ambientales y la preservación del agua como parte de los sistemas ecológicos, enfatizándose, por lo tanto, su uso sostenible, a partir de la Conferencia de Dublín y la Cumbre de la Tierra en 1992.

Lo más reciente a nivel global corresponde a La Asamblea General de Naciones Unidas, que aprobó el 28 de julio de 2010 (7), en su sexagésimo cuarto período de sesiones, una resolución que reconoce al 
agua potable y al saneamiento básico como derecho humano esencial para el pleno disfrute de la vida y de todos los derechos humanos. La resolución fue adoptada a iniciativa de Bolivia, tras 15 años de debates, con el voto favorable de 122 países y 44 abstenciones. La Asamblea de Naciones Unidas (8) se mostró "profundamente preocupada porque aproximadamente 884 millones de personas carecen de acceso al agua potable y más de 2.600 millones de personas no tienen acceso al saneamiento básico, y alarmada porque cada año fallecen aproximadamente 1,5 millones de niños menores de 5 años y se pierden 443 millones de días lectivos a consecuencia de enfermedades relacionadas con el agua y el saneamiento".

Latinoamérica, y Chile en particular, no se escapan de esta problemática. Los cambios climáticos hacen cada vez menos previsible y más fluctuantes las disponibilidades de agua y entre otros, tienden a intensificar los requerimientos de agua para regadío (por aumento de la temperatura ambiental); El Doctor Santibáñez (9) señala en la Revista del Campo, 21 de octubre del 2006: "Se ha registrado un pequeño aumento en la variabilidad del clima en los últimos 30 años respecto de la frecuencia de eventos extremos de hace 60 años. Las precipitaciones son más variables. Se registran más ahora fuera de época, en primavera-verano, que antes; tenemos una frecuencia de eventos del Niño que pareciera ser que es un poco más seguida que antes. Pero los efectos no son al extremo de lo que está pasando en el trópico, donde preocupa mucho más el tema. En Chile la zona más sensible es de Santiago al norte. Los mayores cambios conductuales en los climas se observan entre Copiapó y Santiago, donde se ha producido una disminución en las precipitaciones entre 20 a 30 por ciento durante el siglo que acaba de terminar. Se ha elevado 0,6 a 0,7 grados en la temperatura promedio y aumentaron las temperaturas mínimas, de modo que los inviernos se han hecho más suaves". De esta forma prosigue: "La tecnología de riego va a tener que seguir avanzando hacia el sur y, probablemente, habrá que regular cuencas en las zonas donde ahora no hay grandes obras, con el propósito doble de contar con agua y energía. Ese es un gran desafío que tiene el país: seguir avanzando muy rápidamente para prevenir problemas mayores en el futuro. Aun sin cambios climáticos tiene que hacerlo, simplemente por el crecimiento de su economía”.

\section{De los problemas globales a soluciones locales}

La XV Región de Arica y Parinacota, con casi 200.000 habitantes, posee alrededor de 8.000 ha de riego distribuidas en cuatro cuencas y el sector precordillerano; cada uno de estos sectores con distintas cualidades presentan problemáticas muy peculiares que pasan por la escasa disponibilidad de recurso, la calidad del agua, conflictos de intereses entre distintos sectores, recursos compartidos, desperdicio de recursos, contaminación, recursos no explorados, recursos no dimensionados, nula capacidad de regulación, baja capacidad de manejo, baja capacidad técnica de organizaciones de usuarios, entre otras. En otras palabras no se escapa a la problemática mundial o global en relación al tema.

Sin embargo, siendo la problemática similar a la problemática global, se tiene que tener presente que todas y cada una de las soluciones que se deban aplicar serán de tipo local, ya que es en el mismo territorio y con sus propios actores con los que debe enfrentar la situación.

En este sentido es que cada región, para enfrentar el problema debe hacer los esfuerzos necesarios para generar internamente las capacidades suficientes para abordar de manera asertiva lo complejo del problema.

Las capacidades instaladas en la región deberían tener la misión de apoyar a las organizaciones locales en el inventario de recursos, caracterización y nuevas formas de abastecimiento, uso de tecnologías duras y blandas en el manejo y uso del agua con el menor impacto sobre los ecosistemas, promover normas y leyes, capacitar a los usuarios y sus organizaciones, realizar investigaciones básicas y aplicadas, realizar los análisis socioeconómicos y políticos relacionados, y por ende, desarrollar estrategias locales y propuestas de acción apropiadas con respecto al desarrollo, manejo y preservación de los recursos hídricos.

En este contexto las acciones a emprender debieran abordar una amplia gama de aspectos:

- Inventario de los recursos hídricos

- Caracterización de los sistemas actuales

- Sistematización del funcionamiento de los sistemas

- Investigación y desarrollo de tecnologías de riego sustentables

- Capacitación del sector agrícola

- Capacitación del sector industrial y minero 
- Capacitación población urbana.

- Conceptualización de la dinámica de las distintas cuencas.

- Desarrollo de planes de manejo de cuencas y acuíferos.

- Determinación de las calidades de aguas superficiales y subterráneas.

- Monitoreo y seguimiento de caudales y calidad.

- Evaluación de la vulnerabilidad de los acuíferos.

- Análisis socioeconómicos de los proyectos.

- Evaluaciones económicas de los proyectos.

- Identificación de la interacción agua superficialsubterránea.

- Análisis político, social y económico de las aguas transfronterizas.

- Tecnologías de tratamientos de aguas servidas.

- Sistemas de mejoramientos de aguas.

- Ingeniería de sistemas de riego.

- Desarrollo de cultivos eficientes en el uso del agua.

- Ecología de cuencas.

- Desarrollo de indicadores ecológicos para cuencas.

- Capacitación de dirigentes y autoridades.

- Participación en instancias de planeamiento agrícolas, urbanos e industriales.

- Desarrollo de sistemas de información hidrometeorológicos.

- Optimización de sistemas actuales.

- Catastro de derechos, usos y costumbres.

- Análisis y sistematización de conflictos.

- Análisis de los sistemas tarifarios.

- Modelos hidrológicos predictivos.
- Planes de manejo en períodos de sequía.

- Manejo de afluentes.

- Defensas fluviales.

- Promoción de la cultura del uso sustentable del agua.

- Usos eficientes del agua domiciliaria.

- Manejo y tratamiento de aguas industriales.

- Uso de energías alternativas.

- Establecimiento de líneas base de sectores.

- Determinación de calidad y caudal ecológico de los cuerpos de agua.

- Evaluación ambiental.

- Desarrollo de sistemas de cosecha de agua.

- Técnicas de construcción de infraestructura de captación, regulación y distribución.

- Normativas municipales de construcción de sistemas de agua domiciliaria.

- Normativas municipales de cuidado de cauces.

- Manejo y conservación de humedales.

- Sistemas de mitigación de crecidas.

- Análisis comparativo de normativas y leyes.

- Análisis de propuestas de modificaciones de normas y leyes.

- Búsqueda de nuevas fuentes de recursos hídricos.

- Investigación básica y aplicada.

- Disponer de sistema de alerta sobre problemas de los recursos hídricos, etc.

Se advierte que no es tarea fácil. Nuestra civilización cada vez más populosa y compleja nos obliga a mayores desafíos. Lo que no puede suceder, en cada región, es que no hagamos nada, pensando en que otros vendrán a solucionar nuestros problemas.

\section{Literatura Citada}

(1) Declaración de la conferencia de las Naciones Unidas sobre el Medio Ambiente Humano (Estocolmo, Suecia, 5-16 de junio de 1972).

en: http://www.jmarcano.com/educa/docs/estocolmo.html

(2) Díaz F. F.

2002 Presentación DGA (Dirección General de Aguas Chile) IX Región. Diálogo sobre el Agua. Visión Indígena y acción gubernamental.

(3) Falkenmark, M. and Widstrand, C.

1992 Population and water resources: A delicate balance. Population Bulletin 47(3): 1-36. Nov.

(4) FAO

2005 En:http://www.greenfacts.org/es/recursos-hidricos/ figtableboxes/3.htm. Tabla 4.3 Información sobre la disponibilidad de agua por país (AQUASTAT, FAO 2005)
(5) Hinrichsen, D.; Robey, B. y Upadhyay, U.D.

1997 Soluciones para un Population Reports con escasez de agua del mundo, la Serie M, No 14. Baltimore, Johns Hopkins School of Public Health, Programa de Información sobre Población, diciembre de 1997.

(6) Hitos importantes en: http://hispagua.cedex.es/sites/default/files/hitos_0.pdf

(7) Noticias: http://wapedia.mobi/es/Agua? $\mathrm{p}=2$

(8) ONU, 1987 document A/42/427-Report of the World Commission on Environment and Development: Our Common Future. En: http://www.un-documents.net/wced-ocf.htm

(9) Santibáñez, Fernando

2006 El cambio que viene en el agro (21 / Octubre / 2006) Revista del Campo, El Mercurio, Chile, 